\title{
Acute oral intake of beta-hydroxybutyrate in a pilot study transiently increased its capillary levels in healthy volunteers
}

\begin{abstract}
The popularity of ketogenic diets has led to an increased interest in alternative approaches to inducing and sustaining ketosis. However, published information on the impact of exogenous ketone products in human subjects is lacking. This study aimed to characterize the circulating $\beta$-hydroxybutyrate $(\beta \mathrm{HB})$ response in healthy men and women $(\mathrm{n}=10)$ following acute consumption of $\beta \mathrm{HB}$ salts. In a randomized, cross-over design, participants consumed placebo control or a combination of sodium and calcium $\beta \mathrm{HB}$ salts providing either $11.7 \mathrm{~g}$ (full dose) or $5.85 \mathrm{~g}$ (half dose) of $\beta \mathrm{HB}$, with a wash-out period between intakes. Blood levels of $\beta \mathrm{HB}$ and glucose were measured and vital signs and adverse events were monitored over the following 4hours. Consumption of $11.7 \mathrm{~g} \beta \mathrm{HB}$ led to a significant increase in circulating $\beta \mathrm{HB}$ levels above $1 \mathrm{mmol} / \mathrm{L}$ within 15 minutes compared with the placebo. Intake of the $5.85 \mathrm{~g}$ of $\beta \mathrm{HB}$ led to increases in $\beta \mathrm{HB}$ levels between the full dose and the placebo. The rise in $\beta \mathrm{HB}$ was comparable to that seen in physiological ketogenic situations such as when following a ketogenic diet or periods of fasting, and did not approach the range seen with pathological conditions such as diabetic ketoacidosis. Blood glucose or blood pressure was not adversely impacted during the treatment period. Pulse was seen to modestly but significantly decrease with consumption of the full dose $\beta \mathrm{HB}$. In conclusion, consumption of $11.7 \mathrm{~g} \beta \mathrm{HB}$ can lead to transient increases in capillary $\beta \mathrm{HB}$ in line with thresholds seen in nutritional ketosis conditions.
\end{abstract}

Keywords: ketogenic diet, nutritional ketosis, $\beta$-hydroxybutyrate, exogenous ketone, cross-over trial
Volume 8 Issue 4 - 2018

\author{
Annalouise O'Connor, Jyh-Lurn Chang, \\ Milene Brownlow, Nikhat Contractor \\ Metagenics Inc, USA
}

Correspondence: Annalouise O'Connor, Metagenics Inc. 44th Ave. NW, Gig Harbor,WA, 98332, USA, Tel +253-853-7208, Fax 253-85I-3923, Email annalouiseoconnor@metagenics.com

Received: July 16, 2018 | Published: August 31, 2018

\section{Introduction}

A ketogenic diet refers to a dietary approach that promotes nutritional ketosis by restricting carbohydrates (usually to less than $50 \mathrm{~g}$ per day) and increasing the intake of fat with adequate consumption of protein. With a shortage of available glucose, fat from dietary source or stored adipose deposits is metabolized into ketone bodies [acetoacetate, $\beta$-hydroxybutyrate $(\beta \mathrm{HB})$ and acetone] that can be utilized by the cells for energy. ${ }^{1}$ Systematic reviews and meta-analyses have reported the effectiveness of ketogenic diets in treating intractable epilepsy in adults and children, ${ }^{2,3}$ preventing an increase in appetite on reduced calorie diets, ${ }^{4}$ and achieving longterm bodyweight reduction. ${ }^{5}$ Emerging studies have shown that ketogenic diets may help diabetes management and improve exercise performance.${ }^{6,7}$ However, compliance with a ketogenic diet can be difficult, as many have perceived the diet to be rigid or experienced adverse effects such as gastrointestinal disturbances ${ }^{8,9}$ It has also been reported anecdotally as well as in the literature ${ }^{10}$ that, when following ketogenic diets or very-low-calorie diets (VLCD), there is a short lag time in the increase in circulating ketone bodies in conditions of reduced glucose intake, during which individuals report symptoms described as 'keto flu', including light headedness, irritability, fatigue and hunger. Hence, there has been increased interest in utilizing additional methods to enhance compliance and to facilitate the induction and maintenance of ketosis.

There has been a surge of commercially available products supplying exogenous ketones such as $\beta \mathrm{HB}$ salts. However, the oral response to such $\beta \mathrm{HB}$ salt formulations has been characterized in very few human studies. This report was intended as a proof-of-concept study to determine the circulating $\beta \mathrm{HB}$ concentration in response to acute, oral $\beta \mathrm{HB}$ supplementation. A lower dose was also investigated alongside a no-active placebo control.

\section{Methods and materials}

\section{Study subjects}

Participants were healthy adults aged 21-65y/o with normal body weight, with fasting glucose $<5.55 \mathrm{mmol} / \mathrm{L}(<100 \mathrm{mg} / \mathrm{dL})$ and fasting ketones $<1 \mathrm{mmol} / \mathrm{L}$ at screening. Key exclusion criteria included: currently following a ketogenic diet $(<50 \mathrm{~g}$ carbohydrate per day) or on a weight loss program; use of medications or nutritional supplements which may influence study results; known allergy or hypersensitivity to study products; significant abnormalities in medical history or physical examination; current diagnosis of serious medical conditions; history of drug or alcohol abuse. Study procedures were in accordance with the Declaration of Helsinki and were approved by the Quorum Independent Review Board (Seattle, WA). Written informed consent was obtained from all participants before enrollment.

\section{Study design}

This was a randomized, double-blind, placebo-controlled crossover trial in which $\beta \mathrm{HB}$ concentrations in capillary blood were evaluated following consumption of 2 different doses of $\beta \mathrm{HB}$ salts and a placebo. Each treatment took place on a different study day separated 
by a wash-out period of at least 48 hours and not more than 1 week Participants attended the Functional Medicine Research Center (Gig Harbor, WA) following a 10 hour overnight fast. Participants were advised on composition of evening meal prior to the study days, and were requested to avoid alcohol and physical activity the day before each study visit (Figure 1). The $\beta \mathrm{HB}$ salts or placebo were mixed with $12 \mathrm{oz}$ water and consumed within 5 minutes. Blood was collected immediately prior to consumption, and $0.25,0.5,1,2$ and 4 hours post consumption via finger-stick for glucose and $\beta \mathrm{HB}$ assessments (Figure 1). During the 4 hours post consumption period, all subjects were fasted and were permitted to consume plain water only throughout this time. Body weight was measured at each study visit, and vital signs (pulse, blood pressure, temperature, heart rate) were measured before product consumption and at 4 hours post-consumption. AEs and tolerance information was recorded at the end of each study arm. Circulating glucose and ketone levels were determined using the commercially available monitoring system Precision Xtra ${ }^{\mathrm{TM}}$ (Abbott Diabetes Care Inc., Alameda, CA). The area-under-the-curve (AUC) for $\beta \mathrm{HB}$ was determined using the trapezoidal rule.

\section{Study products}

The full-dose and half-dose study products provided, respectively, $11.7 \mathrm{~g} / \mathrm{serving}$ and $5.85 \mathrm{~g} /$ serving of $\beta \mathrm{HB}$ from calcium and sodium $\beta \mathrm{HB}$ in addition to flavors and excipients to improve product taste tolerability. The placebo control matched flavors and excipients to the full-dose $\beta \mathrm{HB}$ product. $\beta \mathrm{HB}$ was supplied by NNB Nutrition (Frisco, TX).

\section{Statistical analyses}

For normally distributed data, differences between treatment arms were assessed by repeated measures analysis of variance (RMANOVA) with post-hoc testing if significance was identified. Paired t-tests were used to assess within group differences between two time-points. Non-normally distributed data was transformed via log or square root transformation, and normal distribution re-examined. If normality could not be coaxed, Friedman test was used to assess differences between the three treatment groups, and Dunnett's test for multiple comparisons used for post-hoc testing if a significant between-group difference was seen.

\section{Results}

All 10 participants ( 8 women and 2 men; all Caucasians) recruited for this study completed the study. Their mean age (mean \pm SD) was $31.4 \pm 12.0 \mathrm{y} / \mathrm{o}$ and mean BMI was $23.7 \pm 1.3$. Baseline fasting $\beta \mathrm{HB}$ and glucose were $0.17 \pm 0.08 \mathrm{mmol} / \mathrm{L}$ and $5.09 \pm 0.45 \mathrm{mmol} / \mathrm{L}$, respectively. Complete blood count and lab parameters were all within normal range (data not shown). The full-dose product ( $\beta \mathrm{HB}$-full) rapidly increased capillary $\beta \mathrm{HB}$ concentrations during the first $60 \mathrm{~min}$ reaching peak levels of $1.04 \pm 1.63 \mathrm{mmol} / \mathrm{L}$ at $15 \mathrm{~min}$, and returned towards baseline values 2 hours following intake (Table 1$)$. The halfdose product ( $\beta \mathrm{HB}$-half) also resulted in an increase in $\beta \mathrm{HB}$ within the same timeframe, although the overall magnitude of the increase was less pronounced, and the $\beta \mathrm{HB}$ concentrations were not significantly different from placebo.

Differences between groups assessed with Friedman test, with Dunnett's test for multiple comparisons used if overall group differences identified $(\mathrm{p}<0.05)$. Between-treatment differences denoted $\mathrm{as}^{\mathrm{a}, \mathrm{b}, \mathrm{c}}$ with treatments not sharing a letter considered significantly different $(\mathrm{p}<0.05)$. Friedman test revealed a significant between-group difference in $\beta$ HB AUC (Figure 2). Dunnett's post-hoc tests identified a significant difference between $\beta \mathrm{HB}$-full and placebo $(p<0.001)$ but not between $\beta$ HB-full and $\beta$ HB-half $(p=0.35)$. Mean $\beta \mathrm{HB}$ AUC for placebo was lower than $\beta$ HB-half but the betweengroup difference did not reach statistical significance $(p=0.08)$. No difference in blood glucose occurred in any of the treatment groups at any of the time points as assessed by RM-ANOVA (Figure 3). Paired t-tests did not identify any differences between baseline and 4hours for $\beta \mathrm{HB}$-full, $\beta \mathrm{HB}$-half, or for placebo.

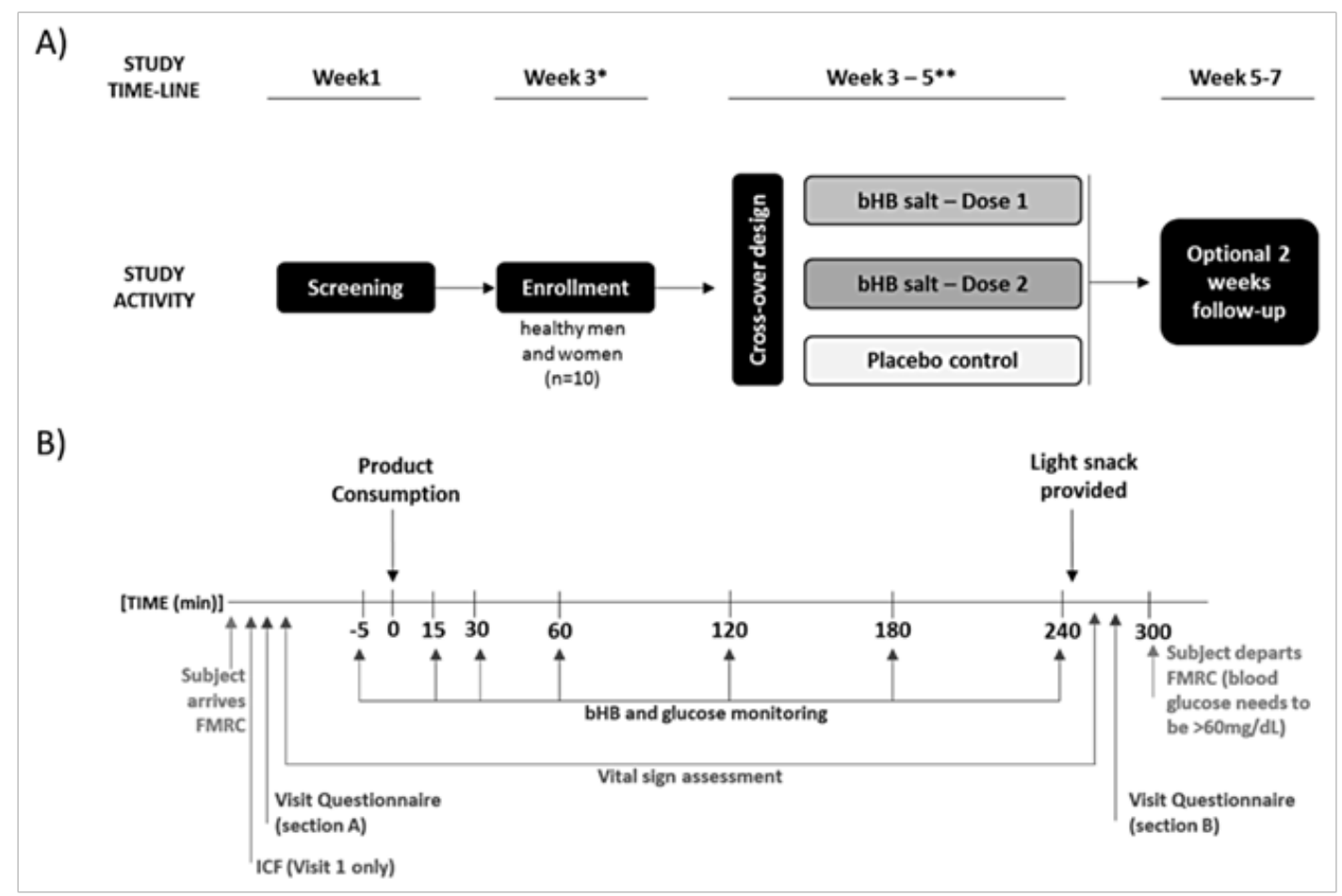

Figure I (A) Study design flow chart and (B) protocol at each clinic visit. 
Table I Circulating $\beta \mathrm{HB}$ concentrations (mean $\pm \mathrm{SE}$ ) from baseline to 4 hours post study treatment consumption

\begin{tabular}{lllll}
\hline & BHB-full & Placebo & $\beta$ HB-half & $\begin{array}{l}\text { P value } \\
\text { (main } \\
\text { effect) }\end{array}$ \\
\hline Baseline & $0.15(0.02)$ & $0.19(0.03)$ & $0.17(0.02)$ & 0.252 \\
\hline I min & I.04(0.52) & $0.17(0.02)^{\mathrm{b}}$ & $0.53(0.1 \mathrm{I})^{\mathrm{bc}}$ & 0.006 \\
\hline $30 \mathrm{~min}$ & $0.69(0.09)^{\mathrm{a}}$ & $0.16(0.02)^{\mathrm{b}}$ & $0.48(0.06)^{\mathrm{ab}}$ & $<0.00 \mathrm{I}$ \\
\hline $60 \mathrm{~min}$ & $0.57(0.04)^{\mathrm{a}}$ & $0.2(0.02)^{\mathrm{b}}$ & $0.3(0.04)^{\mathrm{b}}$ & $<0.00 \mathrm{I}$ \\
\hline I 20min & $0.28(0.03)$ & $0.2(0.02)$ & $0.31(0.08)$ & 0.240 \\
\hline I 80min & $0.22(0.01)$ & $0.27(0.04)$ & $0.21(0.02)$ & 0.239 \\
\hline $240 \mathrm{~min}$ & $0.2(0.03)$ & $0.27(0.05)$ & $0.25(0.02)$ & 0.218 \\
\hline
\end{tabular}

Differences between groups assessed with Friedman test, with Dunnett's test for multiple comparisons used if overall group differences identified ( $\mathrm{p}<0.05$ ). Between-treatment differences denoted as ${ }^{a, b, c}$ with treatments not sharing a letter considered significantly different $(p<0.05)$.

Table 2 Vital signs and body weight pre-and-post study product consumption

\begin{tabular}{|c|c|c|c|c|c|c|}
\hline Product & & Systolic BP (mmHg) & Diastolic BP (mmHg) & $\begin{array}{l}\text { Pulse } \\
\text { (bpm) }\end{array}$ & $\begin{array}{l}\text { Temperature } \\
\text { ( }{ }^{\circ} \text { F) }\end{array}$ & $\begin{array}{l}\text { Weight } \\
\text { (lb) }\end{array}$ \\
\hline \multirow{2}{*}{$\beta$ HB-full } & Pre & I I 2.6 (3.7) & $67.8(3.1)$ & $63.6(4.7)^{\mathrm{a}}$ & $97.7(0.1)^{\mathrm{a}}$ & I 53.7 (4.3) \\
\hline & Post & II 3.7 (3.9) & $67.2(3.8)$ & $56.8(4.0)^{\mathrm{b}}$ & $98.0(0.1)^{b}$ & $153.4(4.3)$ \\
\hline \multirow{2}{*}{ Placebo } & Pre & I | 4.8 (3.0) & $71.4(3.5)$ & $59.8(3.4)$ & $97.7(0.1)^{a}$ & I $52.9(4.4)$ \\
\hline & Post & I I 2.5 (3.7) & 69.7 (3.3) & $58.8(4.0)$ & $98.0(0.1)^{b}$ & I 52.2 (4.4) \\
\hline \multirow{2}{*}{$\beta$ HB-half } & Pre & I I 3.3 (3.7) & $69.8(2.3)$ & $62.7(2.8)$ & $97.8(0.1)^{\mathrm{a}}$ & I 54.2 (4.7) \\
\hline & Post & I I 6.5 (3.6) & $71.2(4.4)$ & $60.0(3.6)$ & $98.1(0.1)^{b}$ & I 53.8 (4.7) \\
\hline
\end{tabular}

Data displayed as mean (SE). Significant within-treatment arm differences between baseline and 4-hours are denoted by ${ }^{\mathrm{a}, \mathrm{b}}$. Time-points not sharing a letter are considered significantly different $(p<0.05)$ as assessed by paired t-tests.

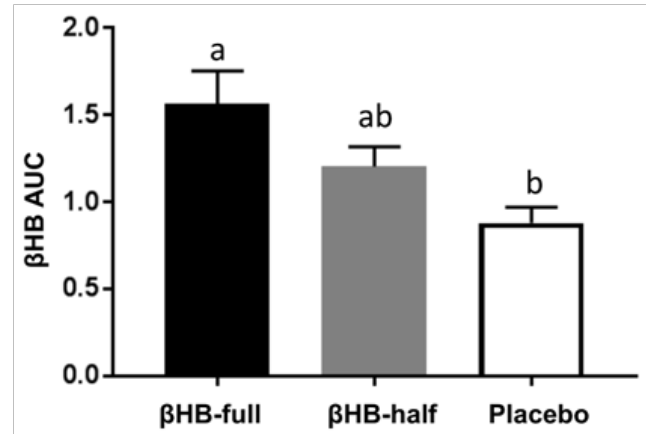

Figure $2 \beta \mathrm{HB}$ area-under-the-curve (AUC; mmol $x$ h). Differences between groups assessed with Friedman test, with Dunnett's test for multiple comparisons used if overall group differences identified. Between-treatment differences denoted $\mathrm{as}^{\mathrm{a}, \mathrm{b}}$ with treatments not sharing a letter considered significantly different $(p<0.05)$. Data expressed as mean \pm SEM.

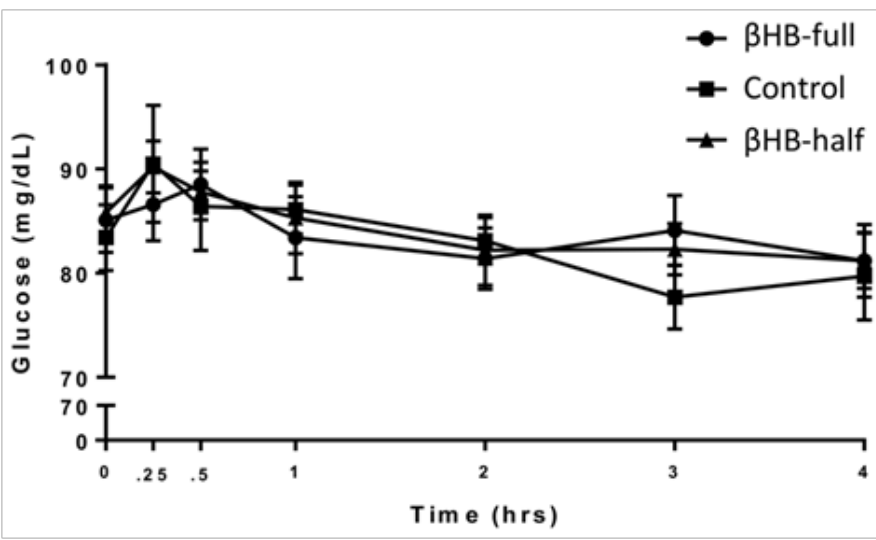

Figure 3 Glucose response from baseline to 4 hours post consumption of $\beta \mathrm{HB}$-full, placebo and $\beta \mathrm{HB}$-half. Data expressed as mean \pm SEM. 
There were no significant differences in any of the vital sign related variables at baseline between the 3 groups as assessed by RMANOVA (Table 2). The small but statistically significant increase in temperature (by a mean of $0.3^{\circ} \mathrm{F}$ ) indicated potential influence of the study environment. There was a statistically significant reduction in pulse with $\beta \mathrm{HB}$-full consumption. One individual reported mild loose stool after taking $\beta \mathrm{HB}$-full and one reported moderate migraine after taking $\beta$ HB-half although the individual had a history of migraine. Data displayed as mean (SE). Significant within-treatment arm differences between baseline and 4-hours are denoted by ${ }^{\mathrm{a}, \mathrm{b}}$. Timepoints not sharing a letter are considered significantly different $(\mathrm{p}<0.05)$ as assessed by paired $\mathrm{t}$-tests.

\section{Discussion}

In this study, consumption of $11.7 \mathrm{~g}$ of $\beta \mathrm{HB}$ led to a significant increase in capillary $\beta \mathrm{HB}$ levels during the first hour compared to control. Increases in $\beta \mathrm{HB}$ with $5.85 \mathrm{~g}$ of $\beta \mathrm{HB}$ (half dose) led to increases in $\beta \mathrm{HB}$ between full dose and placebo control. The magnitude of the rise in $\beta \mathrm{HB}$ was comparable to that seen in physiological ketogenic situations such as when following a ketogenic diet or periods of fasting, ${ }^{11}$ and did not approach the range seen with pathological conditions such as diabetic ketoacidosis. No severe gastrointestinal distress was observed.

When analyzing capillary specimens for $\beta$ HB levels, we noticed data points from one subject (Subject 101) that might be potential outliers. For this subject, the circulating $\beta \mathrm{HB}$ measured $15 \mathrm{~min}$ after the $\beta \mathrm{HB}$-full and $\beta \mathrm{HB}$-half intake was $5.2 \mathrm{mmol} / \mathrm{L}$ and $1.3 \mathrm{mmol} / \mathrm{L}$, respectively, compared with $0.2-0.8 \mathrm{mmol} / \mathrm{L}$ for the other 9 subjects. The circulating $\beta \mathrm{HB}$ measured $4 \mathrm{~h}$ after the placebo intake for was $0.7 \mathrm{mmol} / \mathrm{L}$ for this subject as opposed to $0.2-0.3 \mathrm{mmol} / \mathrm{L}$ for the rest of the group (Supplementary Figure 1). The same statistical analysis excluding this subject revealed that the capillary $\beta \mathrm{HB}$ originally peaked at $15 \mathrm{~min}$ after $\beta \mathrm{HB}$-full intake was reduced to $0.58 \pm 0.23 \mathrm{mmol} / \mathrm{L}$ - and the between-group difference did not reach statistical significance. Instead, it peaked at $30 \mathrm{~min}$ at $0.62 \pm 0.08 \mathrm{mmol} / \mathrm{L}$. The rest of the results including AUC data remained similar (Supplementary Table $1 \&$ Supplementary Figure 2). Whether the cause of the unusual $\beta \mathrm{HB}$ levels seen in this subject was due to hydration status, difference in ketone body metabolism, or measurement error remains to be explored.

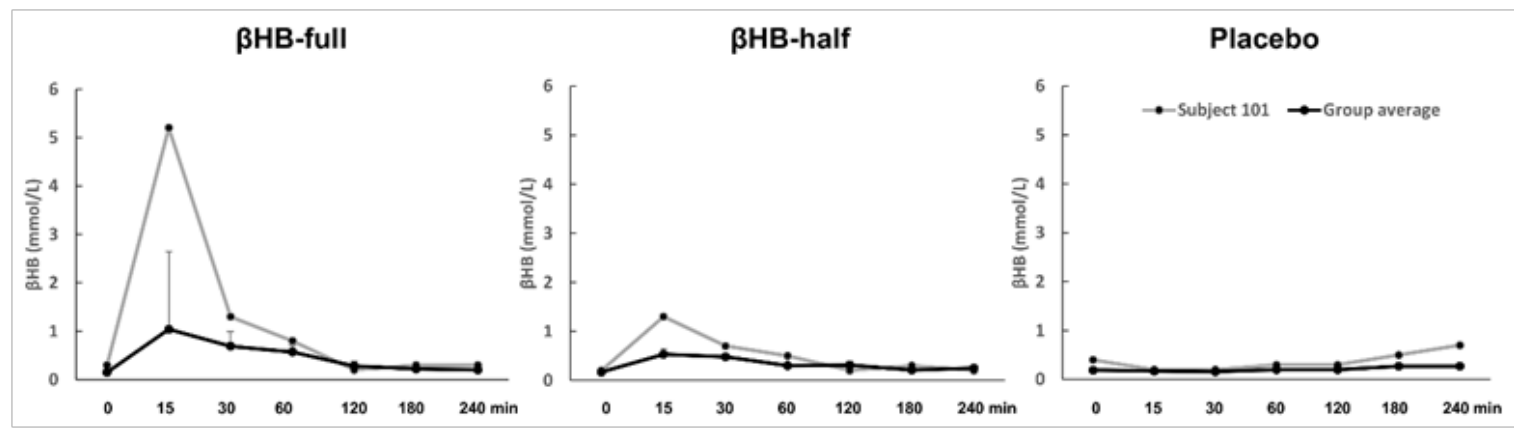

Supplementary Figure I Circulating $\beta H B$ concentrations from Subject I0I vs. group average $(n=10$; mean $+S D)$.

\section{Identification of potential outliers}

Subject I0I's $\beta \mathrm{HB}$ level measured I5 min after the $\beta \mathrm{HB}$-full and $\beta \mathrm{HB}$-half intake was $5.2 \mathrm{mmol} / \mathrm{L}$ and $\mathrm{I} .3 \mathrm{mmol} / \mathrm{L}$, respectively. The circulating $\beta \mathrm{HB}$ measured $4 \mathrm{~h}$ after the placebo intake for was $0.7 \mathrm{mmol} / \mathrm{L}$ for this subject as opposed to $0.2-0.3 \mathrm{mmol} / \mathrm{L}$ for the rest of the group (Supplementary Figure I).

Supplementary Table I Circulating $\beta \mathrm{HB}$ concentrations $(\mathrm{mmol} / \mathrm{L})$ at baseline and 4 hours post study treatment consumption $(\mathrm{n}=9)$

\begin{tabular}{lllll}
\hline & $\beta$ HB-full & Placebo & $\beta$ HB-half & $\begin{array}{l}\text { P value } \\
\text { (main effect) }\end{array}$ \\
\hline Baseline & $0.13(0.02)$ & $0.17(0.02)$ & $0.17(0.02)$ & 0.282 \\
\hline $15 \mathrm{~min}$ & $0.58(0.23)^{\mathrm{a}}$ & $0.17(0.02)^{\mathrm{b}}$ & $0.44(0.23)^{\mathrm{ab}}$ & 0.136 \\
\hline $30 \mathrm{~min}$ & $0.62(0.08)^{\mathrm{a}}$ & $0.16(0.02)^{\mathrm{b}}$ & $0.46(0.06)^{\mathrm{a}}$ & $<0.00 \mathrm{I}$ \\
\hline $60 \mathrm{~min}$ & $0.54(0.04)^{\mathrm{a}}$ & $0.19(0.01)^{\mathrm{b}}$ & $0.28(0.04)^{\mathrm{b}}$ & $<0.00 \mathrm{I}$ \\
\hline $120 \mathrm{~min}$ & $0.29(0.03)$ & $0.19(0.02)$ & $0.32(0.09)$ & 0.234 \\
\hline $180 \mathrm{~min}$ & $0.21(0.01)$ & $0.24(0.03)$ & $0.20(0.02)$ & 0.369 \\
\hline $240 \mathrm{~min}$ & $0.19(0.03)$ & $0.22(0.01)$ & $0.26(0.03)$ & 0.172 \\
\hline
\end{tabular}

Differences between groups assessed with Friedman test, with Dunnett's test for multiple comparisons used if overall group differences identified. Betweengroup differences denoted asa,b,c with treatments not sharing a letter considered significantly different $(p<0.05)$.

\section{Statistical analysis omitting $\beta \mathrm{HB}$ data from Subject 10 I}

$\beta \mathrm{HB}$-full resulted in a rapid increase in capillary $\beta \mathrm{HB}$ concentrations within 60 minutes, with return towards baseline values thereafter. $\beta$ HB-half also resulted in an increase in $\beta \mathrm{HB}$ within the same time-frame, although the overall magnitude of the increase was less pronounced (Supplementary Table I). 


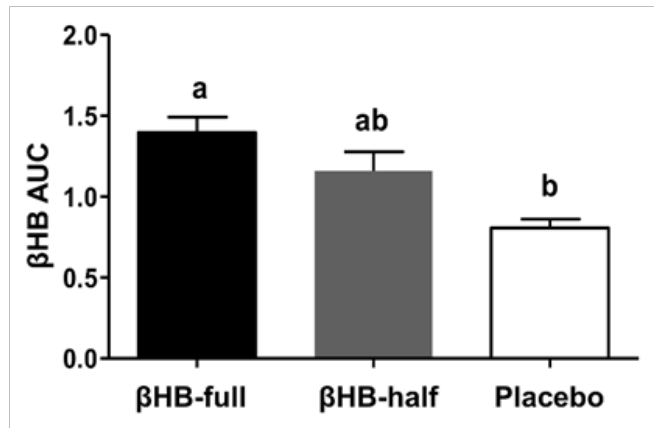

Supplementary Figure $2 \beta \mathrm{HB}$ area-under-the-curve (AUC; mmol $\times \mathrm{h}$ ). Between-treatment differences denoted as a, $b$ with treatments not sharing a letter considered significantly different $(\mathrm{p}<0.05)$. Data expressed as mean \pm SEM. $\beta$ HB AUC data omitting Subject I0 I

Friedman test revealed a significant between-group difference in $\beta H B$ AUC (Supplementary Figure 2). Dunnett's post-hoc tests identified a significant difference between $\beta \mathrm{HB}$-full and placebo control $(p<0.00 \mathrm{I})$, with no significantly difference between $\beta$ HB-full and $\beta$ HB-half $(p=0.095)$ or between $\beta$ HB-half and control $(p=0.06)$.

During our manuscript preparation, two human studies that included an objective similar to ours were published. ${ }^{12,13}$ In one of the experiments published by Stubbs et al., ${ }^{12} 15$ participants ingested a dose of a combination of sodium and potassium $\beta \mathrm{HB}$ providing $\sim 12 \mathrm{~g} \beta \mathrm{HB}$, similar to our full dose, and the circulating $\beta \mathrm{HB}$ levels reached $1.0 \pm 0.1 \mathrm{mmol} / \mathrm{L}($ mean $\pm \mathrm{SE}$ ) during the first hour. Although in our study the peak concentration occurred sooner; at 15minutes (or at 30minutes excluding data from the potential outlier), our AUC data were similar to theirs, and the $\beta \mathrm{HB}$ levels returned to baseline after 2 hours in both studies. In the second publication, ${ }^{13} 6$ healthy adults ingested one dose of a combination of sodium and calcium $\beta \mathrm{HB}$ salts providing $30-57.5 \mathrm{~g}$ depending on their bodyweight, but one subject immediately dropped out due to severe vomiting. The circulating $\beta \mathrm{HB}$ levels increased slowly and peaked at 2.5 hour post-consumption at $0.598 \pm 0.300 \mathrm{mmol} / \mathrm{L}$ (mean $\pm \mathrm{SD}$ ), suggesting a different absorption rate. Glucose levels were not affected. However, multiple subjects experienced mild-to-moderate gastrointestinal discomfort after ingestion, indicating a dose level unsuitable for long-term application.

In our study, blood glucose or blood pressure were not adversely impacted by the $\beta \mathrm{HB}$ product, which is a relevant outcome considering that the subjects consumed the product while fasted and had fasting glucose levels within normal range $(<5.55 \mathrm{mmol} / \mathrm{L})$. This lack of acute ketone-inducing hypoglycemia is corroborated in the study by Stubbs et al. ${ }^{12}$ Another study by Stubbs et al. ${ }^{14}$ demonstrated that acute intake of exogenous ketone ester reduced plasma ghrelin levels and suppressed appetite in healthy individuals. Regarding concerns that exogenous ketone products may potentially increase mineral intakes, Stubbs et al. found that a high dose keto salt $(\sim 24 \mathrm{~g})$ significantly decreased blood potassium (below reference range for 1hour) and increased sodium but it remained within reference range. ${ }^{12}$ In comparison, the doses of keto salt in our study were much lower, although we used a sodium/ calcium mixture instead of potassium/sodium mixture. Whether the mineral content in the exogenous ketone product results in any longterm health impact requires further investigation.

\section{Conclusion}

Our study provides evidence of acute-dose efficacy and safety of an exogenous $\beta \mathrm{HB}$ salt product. Exogenous ketone drinks may help support a ketogenic lifestyle-without the need for strict dietary changes - by facilitating the keto-adaptation process and by increasing $\beta \mathrm{HB}$ concentration to therapeutic levels in individuals already following a ketogenic diet. However, research on the safety and efficacy of exogenous ketones in humans is still scarce. It is of particular interest to better understand nutritional interventions that can support and facilitate the adoption of a ketogenic lifestyle, in addition to considering whether exogenous ketosis can elicit similar benefits observed with nutritional or endogenous ketosis.

\section{Funding}

The study was funded by Metagenics, Inc.

\section{Acknowledgment}

We thank Kimberly Koch for technical and clinical assistance.

\section{Conflict of interests}

All authors are employees of Metagenics, Inc.

\section{References}

1. Fukao T, Lopaschuk GD, Mitchell GA. Pathways and control of ketone body metabolism: on the fringe of lipid biochemistry. Prostaglandins Leukot Essent Fatty Acids. 2004;70(3):243-251.

2. Ye F, Li XJ, Jiang WL, et al. Efficacy of and patient compliance with a ketogenic diet in adults with intractable epilepsy: a meta-analysis. $J$ Clin Neurol. 2015;11(1):26-31.

3. Rezaei S, Abdurahman AA, Saghazadeh A, et al. Short-term and long-term efficacy of classical ketogenic diet and modified Atkins diet in children and adolescents with epilepsy: A systematic review and meta-analysis. Nutr Neurosci. 2017:1-18.

4. Gibson AA, Seimon RV, Lee CM, et al. Do ketogenic diets really suppress appetite? A systematic review and meta-analysis. Obes Rev. 2015;16(1):64-76.

5. Bueno NB, de Melo IS, de Oliveira SL, et al. Very-low-carbohydrate ketogenic diet v. low-fat diet for long-term weight loss: a meta-analysis of randomised controlled trials. Br J Nutr. 2013;110(7):1178-1187.

6. Feinman RD, Pogozelski WK, Astrup A, et al. Dietary carbohydrate restriction as the first approach in diabetes management: critical review and evidence base. Nutrition. 2015;31(1):1-13.

7. Volek JS, Noakes T, Phinney SD. Rethinking fat as a fuel for endurance exercise. Eur J Sport Sci. 2015;15(1):13-20.

8. Wheless JW. The ketogenic diet: an effective medical therapy with side effects. J Child Neurol. 2001;16(9):633-635.

9. Cai QY, Zhou ZJ, Luo R, et al. Safety and tolerability of the ketogenic diet used for the treatment of refractory childhood epilepsy: a systematic review of published prospective studies. World J Pediatr. 2017;13(6):528-536.

10. Krotkiewski M. Value of VLCD supplementation with medium chain triglycerides. Int J Obes Relat Metab Disord. 2001;25(9):1393-1400.

11. Cahill GF. Fuel Metabolism in Starvation. Annu Rev Nutr. 2006;26(1):122.

12. Stubbs BJ, Cox PJ, Evans RD, et al. On the Metabolism of Exogenous Ketones in Humans. Front Physiol. 2017;8(848).

13. Fischer T, Och U, Klawon I, et al. Effect of a sodium and calcium DL-beta-hydroxybutyrate salt in healthy adults. Journal of Nutrition and Metabolism. 2018;2018:8.

14. Stubbs BJ, Cox PJ, Evans RD, et al. A Ketone Ester Drink Lowers Human Ghrelin and Appetite. Obesity (Silver Spring). 2018;26(2):269273. 